\title{
Omega-6 polyunsaturated fatty acids: Is a broad cholesterol-lowering health claim appropriate?
}

\author{
Richard P. Bazinet PhD, Michael W.A. Chu MD MEd
}

Competing interests: Richard Bazinet has received research funding from Bunge Ltd., travel support from Unilever and consultant fees from Kraft Foods Inc. Michael Chu has received speaker fees and laboratory research support from Medtronic of Canada Ltd. and Edwards

Lifesciences; he has also received laboratory research support from NeoChord Inc.

This article has been peer reviewed.

Correspondence to: Richard P. Bazinet, richard.bazinet@utoronto.ca

CMAJ 2014. DOI:10.1503 /cmaj.130253
I n 2009, Health Canada's Food Directorate received a submission from industry requesting approval to use a claim of reduced disease risk on labels of vegetable oils and foods containing vegetable oils. ${ }^{1}$ The health claim was related to the replacement of saturated animal fats with unsaturated fats, including polyunsaturated fatty acids, for "a reduced risk of heart disease by lowering blood cholesterol levels." Health Canada's Food Directorate reviewed the available published literature and concluded that, when saturated fats were replaced with polyunsaturated fatty acids, there was a statistically significant reduction in total and low-density lipoprotein (LDL) cholesterol, that the result was a reduction in the risk of coronary artery disease and that the therapeutic health claim (Box 1) was substantiated by scientific evidence and therefore was valid and "relevant to the Canadian population," given nearly half of Canadians have hyperlipidemia. ${ }^{1}$

Careful evaluation of recent evidence, however, suggests that allowing a health claim for vegetable oils rich in omega- 6 linoleic acid but relatively poor in omega- $3 \alpha$-linolenic acid may not be warranted (Table 1). ${ }^{2-6}$

\section{- KEY POINTS}

- The replacement of dietary saturated fats with some, but not all polyunsaturated fatty acids reduces serum cholesterol levels and the risk of heart disease.

- New analyses suggest that replacing saturated animal fats with linoleic acid, an omega- 6 polyunsaturated fatty acid, lowers serum cholesterol levels but increases the risk of death from coronary artery disease.

- Health Canada's Food Directorate should reconsider the health claim that omega- 6 polyunsaturated fatty acids reduce the risk of heart disease by lowering blood cholesterol levels.

\section{What is known about the cardiovascular health benefits of polyunsaturated fatty acids?}

Polyunsaturated fatty acids are fatty acids with 2 or more double bonds in the carbon chain. The most nutritionally relevant are the omega- 3 fatty acids $(\alpha$-linolenic acid, eicosapentaenoic acid and docosahexaenoic acid) and the omega-6 fatty acids (linoleic acid, $\boldsymbol{\gamma}$-linolenic acid and arachidonic acid) (Figure 1, Table 2). The health claim refers to polyunsaturated fatty acids derived from vegetable oils, largely omega- 6 linoleic acid and omega- $3 \alpha$-linolenic acid, and not to those derived from fish oils, such as eicosapentaenoic acid and docosahexaenoic acid (Figure 1, Table 2). Several large randomized controlled trials have shown fish oils to be associated with a decreased risk of cardiovascular events. ${ }^{7,8}$ However, LDL and total cholesterol levels were either increased ${ }^{7}$ or not different from those in controls, ${ }^{8}$ which may explain why omega-3 fatty acids from fish oils are exempt from the cholesterol-lowering health claim.

Neither omega- 3 nor omega- 6 fatty acids can be synthesized de novo in mammals and are considered nutritionally essential. ${ }^{9,10}$ The nutritional requirement of linoleic acid is not fully agreed upon, possibly because most of the animal modelling and human cases of linoleic acid deficiency were not specific to linoleic acid and included the deficiency of multiple fatty acids, sometimes referred to as essential fatty acid deficiency. ${ }^{11}$ The nutritional requirement of linoleic acid has been estimated to be as high as $2 \%$ of energy but is likely closer to $0.4 \%$ of energy. ${ }^{11}$ Nevertheless, the current dietary intake of linoleic acid by Canadians is about $5 \%-6 \%$ of energy, and Health Canada reports no concerns regarding low intake levels of 
linoleic acid in Canada. ${ }^{12}$ Whereas the US Institute of Medicine has determined adequate intakes for both linoleic acid and $\alpha$-linolenic acid, there are no formal requirements for eicosapentaenoic acid or docosahexaenoic acid, ${ }^{9}$ in part because they can be synthesized from $\alpha$-linolenic acid. ${ }^{9,10}$ As with linoleic acid, intakes of $\alpha$-linolenic acid in Canada are generally considered to be adequate. ${ }^{12}$

\section{What high-quality evidence supports the health claim?}

Consistent with the health claim, a 2003 metaanalysis supported the finding that substitution of saturated fatty acids with vegetable oils rich in polyunsaturated fatty acids, including linoleic acid, lowers serum total and LDL cholesterol levels. ${ }^{2}$ However, the effect of this substitution on the risk of coronary artery disease is not clear. In 2010, a meta-analysis of clinical trials, cited by Health Canada's Food Directorate, ${ }^{1}$ reported that substituting vegetable oils rich in polyunsaturated fatty acids for saturated fat lowers the risk of coronary artery disease (risk ratio [RR] 0.90, 95\% confidence interval [CI] 0.83-0.97, for each change of $5 \%$ energy). ${ }^{13}$ The American Heart Association and the media ${ }^{14}$ have ascribed the noted beneficial effects to linoleic acid, at least in part. ${ }^{15}$ The American Heart Association stated that "higher intakes [of linoleic acid] appear to be safe and may be even more beneficial (as part of a low-saturated-fat, low-cholesterol diet)." 15 This recommendation is shared by some, but not all, nutritional guidelines. ${ }^{16}$

A closer look at the composition of the vegetable oils used in the individual clinical trials raises doubt as to whether the protective effects can be attributed to linoleic acid. In 2010, Ramsden and colleagues performed a detailed analysis of the sources and fatty-acid composition of dietary fats that were exchanged in the clinical trials. ${ }^{17}$ They noted that rates of nonfatal myocardial infarction and death from coronary artery disease were $22 \%$ lower (RR 0.78 , 95\% CI 0.65 0.93 ) when oils containing a mix of linoleic and omega-3 fatty acids were substituted. However, the rates of nonfatal myocardial infarction and death from coronary artery disease were not significantly lower (RR 1.13, 95\% CI 0.84-1.53) when oils made up mostly of linoleic acid but relatively low in $\alpha$-linolenic acid were substituted. As discussed by Ramsden and colleagues and others, ${ }^{17,18}$ it seems unlikely that the beneficial effects of vegetable oils can be attributed to linoleic acid.

Soybean oil (mixed linoleic and $\alpha$-linolenic acids; Table 3 ) was the most commonly substi- tuted oil in the collective group of studies that showed a protective effect. Corn and safflower oil (oils rich in linoleic acid; Table 3), which contain virtually no $\alpha$-linolenic acid, were not associated with a benefit. A test of heterogeneity between oils rich in linoleic acid and those containing mixed polyunsaturated fatty acids was statistically different for nonfatal myocardial infarction and death from coronary artery disease $(p=0.02) .{ }^{17}$

Box 1: The following statements can be made in the labelling and advertising of food products that meet the qualifying criteria'

Primary statement:

"Replacing saturated fats with polyunsaturated and monounsaturated fats (from vegetable oils) helps lower/reduce cholesterol. [Statement that the food is reduced or lower in saturated fat as defined in items 20 and 21 , respectively, in the table following section B.01.513 of the Food and Drug Regulations] (and is a source of omega-3/omega- 6 polyunsaturated fat)."

Examples:

If the food is a vegetable oil:

"Replacing saturated fats with polyunsaturated and monounsaturated fats from vegetable oils helps lower cholesterol. 2 teaspoons $(10 \mathrm{~mL})$ of this blend of corn and canola oil contains $84 \%$ less saturated fat than 2 teaspoons $(10 \mathrm{~g})$ of butter."

If the food is made with a vegetable oil or a blend of vegetable oils: "Replacing saturated fats with polyunsaturated and monounsaturated fats from vegetable oils helps lower cholesterol. This blueberry muffin $(55 \mathrm{~g})$ is made with canola oil, contains $25 \%$ less saturated fat than our regular blueberry muffin $(60 \mathrm{~g})$ and is a source of omega-3 polyunsaturated fat."

The following additional statement could be used in letters up to the same size and prominence as those of the primary statement:

"High cholesterol is a risk factor for heart disease."

Table 1: Summary of common sources of evidence relating omega- 6 linoleic acid or a mixture of linoleic and omega- $3 \alpha$-linolenic acids to cardiovascular health benefits

Fatty acid; evidence of link to health benefit

\begin{tabular}{|c|c|c|}
\hline \multirow[b]{2}{*}{ Health benefit } & \multicolumn{2}{|c|}{ Fatty acid; evidence of link to health benefit } \\
\hline & $\begin{array}{l}\text { Omega-6 } \\
\text { linoleic acid }\end{array}$ & $\begin{array}{l}\text { Mix of linoleic and } \\
\alpha \text {-linolenic acids }\end{array}$ \\
\hline Lowers cholesterol & Yes $^{2}$ & Yes $^{2}$ \\
\hline $\begin{array}{l}\text { Lowers risk of coronary } \\
\text { artery disease in prospective } \\
\text { cohort studies* }\end{array}$ & Unclear 3,4 & $\mathrm{Yes}^{3,4}$ \\
\hline $\begin{array}{l}\text { Lowers risk of coronary } \\
\text { artery disease in clinical trials }\end{array}$ & $\mathrm{No}^{5}+$ & Yes $^{5}$ \\
\hline Health claim allowed & Yes & Yes \\
\hline \multicolumn{3}{|c|}{$\begin{array}{l}\text { *Whereas mixed polyunsaturated fatty acids containing omega- } 3 \alpha \text {-linolenic acid have been } \\
\text { found to be protective against coronary artery disease in prosective trials, one report that } \\
\text { attempted to isolate linoleic acid's effect found no protective effect. }{ }^{4} \text { Nevertheless, it is } \\
\text { unclear whether the methodology used in prospective cohorts can isolate linoleic acid from } \\
\alpha \text {-linolenic acid. }{ }^{6} \\
\text { tBoth the Sydney Diet Heart Study and a recent meta-analysis reported an increased risk of } \\
\text { death from coronary artery disease, although the risk was not statistically significant in the } \\
\text { meta-analysis (hazard ratio for death from coronary artery disease } 1.33,95 \% \text { confidence } \\
\text { interval } 0.99-1.79) .{ }^{5}\end{array}$} \\
\hline
\end{tabular}


This finding supports the idea that oils rich in linoleic acid should not be grouped with those containing mixed polyunsaturated fatty acids. However, because relatively few studies examined linoleic acid specifically (3 clinical trials), ${ }^{17}$ it is not possible to draw strong conclusions.

Ramsden and colleagues recently completed a detailed reanalysis of recovered data from the Sydney Diet Heart Study, in which the intervention group replaced saturated fat with sources of safflower oil or safflower oil margarine (rich in omega- 6 linoleic acid but low in omega- $3 \alpha$ linoleic acid). ${ }^{5}$ They found that the intervention group had serum cholesterol levels that were significantly decreased (by about 8\%-13\%) relative to baseline and the control group, which is consistent with the health claim. However, the interven- tion group had significantly higher rates of death than the control group (all cause $17.6 \%$ v. $11.8 \%$ [hazard ratio (HR) 1.62, 95\% CI 1.00-2.64]; cardiovascular disease $17.2 \%$ v. $11.0 \%$ [HR 1.70, 95\% CI 1.03-2.80]; and coronary artery disease $16.3 \%$ v. $10.1 \%$ [HR $1.74,95 \%$ CI 1.04-2.92]).

After updating their previous meta-analysis with the recovered data, Ramsden and colleagues reported that the rate of death from coronary artery disease was not significantly lower when saturated fats were replaced with oils containing mixed polyunsaturated fatty acids (HR $0.81,95 \%$ CI $0.64-1.03)$; however, the rate of cardiovascular-related death was significantly reduced (HR 0.79, 95\% CI 0.63-0.99). ${ }^{5}$ They also confirmed that the use of oils rich in linoleic acid was not associated with a significant de-

\section{(A) omega-6 \\ linoleic acid \\ $18: 2 n-6$}

Omega-6 double bond

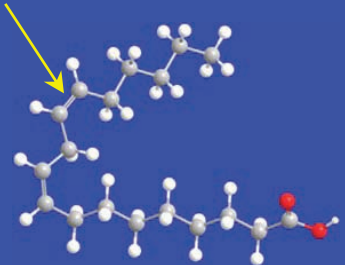

(D) omega-6

arachidonic acid

20:4n-6

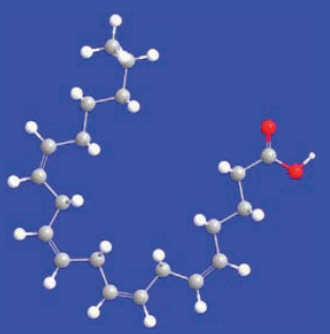

(B) omega-3

$\alpha$-linolenic acid

$18: 3 n-3$
(C) omega-9

oleic acid

18:1n-9

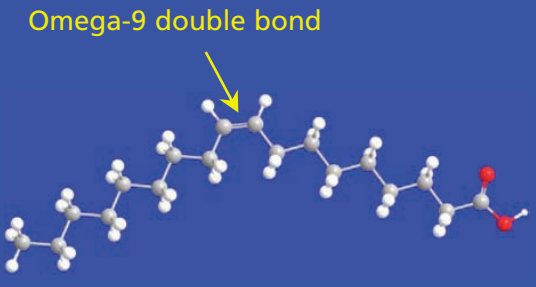

(F) omega-9

mead acid

20:3n-9

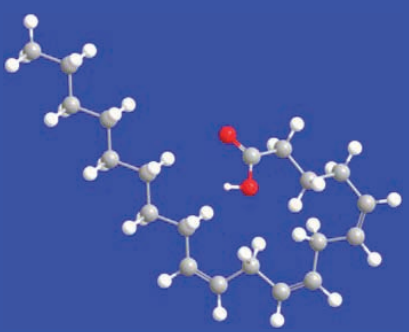

Figure 1: The structure of selected unsaturated fatty acids. (A) Linoleic acid (18:2n-6) is 18 carbons long with 2 double bonds. It is in the omega- 6 family of fatty acids because the first double bond is on the sixth carbon from the methyl terminal end. (B) $\alpha$-Linolenic acid $(18: 3 n-3)$ is also 18 carbons long, but with 3 double bonds. It is in the omega- 3 family because the first double bond is on the third carbon from the methyl terminal end. Both omega- 6 and omega-3 fatty acids cannot be synthesized de novo in mammals and therefore are considered nutritionally essential. (C) Oleic acid is an omega-9 fatty acid that can be obtained from the diet or synthesized de novo. (D) Arachidonic acid is an omega-6 fatty acid that can be obtained from the diet or from the metabolism of linoleic acid. (E) Eicosapentaenoic acid is an omega-3 fatty acid that can be synthesized from $\alpha$-linolenic acid or obtained from the diet, especially from fish oils. Although the term "polyunsaturated fatty acid" generally refers to the omega- 6 and omega-3 families, mead acid (F) is technically an omega-9 polyunsaturated fatty acid. Some confusion arises because linoleic acid is quantitatively the most abundant dietary omega- 6 polyunsaturated fatty acid, and thus the terms "linoleic acid" and "omega- 6 polyunsaturated fatty acid" or even "polyunsaturated fatty acids" are sometimes used interchangeably. Furthermore, chemists commonly number carbons from the carboxylic acid (red) end, whereas nutritionists number them from the omega (methyl) end. 
crease in death from coronary artery disease (HR $1.33,95 \%$ CI $0.99-1.79$ ) or from cardiovascular disease (HR 1.27, 95\% CI 0.98-1.65). Although the mechanism by which replacing saturated fats with omega-6 linoleic acid would increase the risk of death from coronary artery disease is unclear, the authors noted that oxidized linoleic acid is the most abundant oxidized fatty acid in lipoproteins. Secondary analyses confirmed that the detrimental effects of linoleic acid were seen in participants who were smokers and those who consumed alcohol, people likely to be under increased oxidative stress.

The re-evaluation of data from the Sydney Diet Heart Study had limitations. ${ }^{5}$ The participants were survivors of a recent coronary event, about $70 \%$ were smokers, and the study may have been underpowered. Furthermore, the exact composition of fatty acids in the substitute margarine was not known, and it may have included trans fatty acids. However, the intervention resulted in decreased serum cholesterol levels, and the effects remained significant after theoretical adjustments for trans fatty acids. The updated meta-analysis also had limitations. ${ }^{5}$ Several

Table 2: Selected polyunsaturated fatty acids

\begin{tabular}{|lc|}
\hline Fatty acid; common name & Short hand \\
\hline Omega-3 & \\
\hline$\alpha$-Linolenic acid & $18: 3 n-3$ \\
\hline Eicosapentaenoic acid & $20: 5 n-3$ \\
\hline Docosahexaenoic acid & $22: 6 n-3$ \\
\hline Omega-6 & \\
\hline Linoleic acid & $18: 2 n-6$ \\
\hline -Linolenic acid & $18: 3 n-6$ \\
\hline Arachidonic acid & $20: 4 n-6$ \\
\hline
\end{tabular}

of the clinical trials involved patients with psychiatric disorders or with existing coronary artery disease. Furthermore, multiple fats were replaced in the control groups, and different doses and sources of oils were used in the intervention groups.

Notwithstanding these limitations, Ramsden and colleagues' reanalysis has raised several important questions. Is it fair to say that there was a trend toward omega- 6 linoleic acid increasing the risk of death from coronary artery disease when the $95 \% \mathrm{CI}$ in the meta-analysis was $0.99-1.79$, overlapping 1 ? Should oils that are rich in omega- 6 linoleic acid and that lower cholesterol be granted a health claim that implies a reduction in risk of coronary artery disease based on these data? Was it the omega- $3 \alpha$ linolenic acid in the oils with mixed polyunsaturated fatty acids that was protective against death from cardiovascular disease? Despite the limitations of recent studies, it seems unlikely, however, that additional secondary analyses or new interpretations of these datasets will lead to the conclusion that increasing intake of omega- 6 linoleic acid will decrease the risk of death from coronary artery disease.

\section{What have epidemiologic studies shown?}

When considering evidence for the health claim, inclusion of prospective cohort studies that have examined omega- 6 linoleic acid and coronary artery disease is probably warranted given the limitations of the clinical trials. A summary of the evidence provided from prospective cohort studies is shown in Table 1.

In observational studies, foods are usually identified and quantified using a food frequency

Table 3: Composition of common vegetable oils

\begin{tabular}{|c|c|c|c|c|c|}
\hline \multirow[b]{2}{*}{ Vegetable oil } & \multicolumn{5}{|c|}{ Fatty acid, $\mathrm{g} / 100 \mathrm{~g}$ of oil* } \\
\hline & Saturated & Monounsaturated & Linoleic (omega-6) & $\alpha$-Linolenic (omega-3) & Linoleic: $\alpha$-linolenic ratio \\
\hline Canola & 7.4 & 61.7 & 19.0 & 9.1 & 2.1 \\
\hline Canola high in oleic acid & 7.4 & 78.7 & 7.8 & 2.6 & 3.0 \\
\hline Soybean & 15.7 & 22.7 & 50.9 & 6.8 & 7.5 \\
\hline Corn & 12.9 & 27.6 & 27.3 & 1.1 & 24.8 \\
\hline Safflower & 6.2 & 14.4 & 74.6 & $<0.1$ & $>100$ \\
\hline Sunflower & 10.3 & 19.5 & 65.7 & $<0.1$ & $>100$ \\
\hline Olive & 13.8 & 72.9 & 9.7 & 0.8 & 12.1 \\
\hline
\end{tabular}


questionnaire and are matched to databases with the nutrient composition of the foods. The assessment of dietary intakes of omega- 6 linoleic acid and omega- $3 \alpha$-linolenic acid from food frequency questionnaires has been criticized. ${ }^{6}$ In addition, dietary intake of $\alpha$-linolenic acid as measured by food frequency questionnaires has been found to correlate weakly with plasma phospholipid, cholesteryl ester and adipose levels (Pearson correlation coefficients 0.15 and 0.31 , and Spearman correlation coefficient 0.33 , respectively; $p<0.05$ ) in some food frequency questionnaires, ${ }^{21,22}$ and to have no significant correlation with adipose, erythrocyte or plasma $\alpha$ linolenic acid levels (Spearman correlation coefficients $0.01-0.12$ for adipose and $0.17-0.23$ for blood measures; $p>0.05$ ) in other food frequency questionnaires. ${ }^{21,23}$ However, dietary intake of both linoleic and $\alpha$-linolenic acids as measured by food frequency questionnaires correlate moderately with each other (Spearman correlation coefficient $0.49, p<0.001){ }^{24}$ Thus, it is unclear whether the methodologic features of food frequency questionnaires can isolate dietary linoleic acid from $\alpha$-linolenic acid.

Dietary sources of omega- 6 linoleic acid measured by food frequency questionnaires are not reported in most prospective studies examining coronary artery disease. In one report from the Nurses Health Cohort, in which increasing intake of linoleic acid was associated with an increased risk of clinical depression, ${ }^{25}$ separately published food sources of linoleic acid ${ }^{26}$ were mayonnaise or other creamy vinaigrettes $(14.6 \%$ of total dietary linoleic acid), margarine (9.8\%), oil and vinegar salad dressing $(9.1 \%)$, fried food at home and away $(7.1 \%)$, chips $(5.5 \%)$, nuts $(4.7 \%)$ and peanut butter $(4.1 \%) .{ }^{26}$ It is unclear whether any of these foods were associated with the protective effects against coronary artery disease ascribed to linoleic acid observed in some, ${ }^{3}$ but not all, ${ }^{4}$ of the prospective cohorts.

\section{What are the trends in oil usage in Canada?}

Currently, relatively pure dietary oil sources of omega-6 linoleic acid make up only 5\% of oils in the Canadian diet; canola oil and soybean oil account for about $55 \%$ and $25 \%$ of dietary intake, respectively, ${ }^{27}$ both of which contain mixed linoleic and $\alpha$-linolenic acids (Table 3 ). An emerging body of literature supports the hypothesis that omega- $3 \alpha$-linolenic acid may reduce the risk of coronary artery disease, ${ }^{28}$ but evidence from clinical trials that have attempted to isolate $\alpha$-linolenic acid is limited. ${ }^{29}$ In Canada and elsewhere, traditional canola and soybean oils are being replaced with versions high in oleic acid (an omega-9 monounsaturated fatty acid), at the expense of omega- 6 linoleic acid and especially omega- $3 \alpha$-linolenic acid. The effect of oils high in oleic acid on the risk of cardiovascular disease has yet to be tested in randomized or prospective trials.

\section{Conclusion}

In summary, much confusion has arisen from unclear nomenclature with regard to polyunsaturated fatty acids and the lack of reporting of food sources in prospective cohorts. Data from prospective cohort studies and randomized controlled trials generally support the replacement of saturated fats with mixed polyunsaturated fatty acids to reduce the risk of death from coronary artery disease. However, it is unclear whether oils rich in omega- 6 linoleic acid but low in omega- $3 \alpha$-linolenic acid also reduce this risk. We suggest that the health claim be modified such that foods rich in omega- 6 linoleic acid but poor in omega- $3 \alpha$-linolenic acid be excluded. Future research is needed to examine the dietary methodologies used to measure polyunsaturated fatty acid intakes in observational studies and the metabolism of polyunsaturated fatty acids, as well as their role in coronary artery disease and other diseases.

\section{References}

1. Health Canada. Summary of Health Canada's assessment of a health claim about the replacement of saturated fat with monoand polyunsaturated fat and blood cholesterol lowering. Ottawa (ON): Health Canada; 2012. Available: www.hc-sc.gc.ca/fn-an /label-etiquet/claims-reclam/assess-evalu/sat-mono-poly-fat-gras -eng.php (accessed 2013 Feb. 7).

2. Mensink RP, Zock PL, Kester AD, et al. Effects of dietary fatty acids and carbohydrates on the ratio of serum total to HDL cholesterol and on serum lipids and apolipoproteins: a meta-analysis of 60 controlled trials. Am J Clin Nutr 2003;77:1146-55.

3. Hu FB, Stampfer MJ, Manson JE, et al. Dietary fat intake and the risk of coronary heart disease in women. N Engl J Med 1997;337: 1491-9.

4. Mozaffarian D, Ascherio A, Hu FB, et al. Interplay between different polyunsaturated fatty acids and risk of coronary heart disease in men. Circulation 2005;111:157-64.

5. Ramsden CE, Zamora D, Leelarthaepin B, et al. Use of dietary linoleic acid for secondary prevention of coronary heart disease and death: evaluation of recovered data from the Sydney Diet Heart Study and updated meta-analysis. BMJ 2013;346: e8707.

6. Ramsden CE, Hibbeln JR, Majchrzak-Hong SF. All PUFAs are not created equal: absence of CHD benefit specific to linoleic acid in randomized controlled trials and prospective observational cohorts. World Rev Nutr Diet 2011;102:30-43.

7. Dietary supplementation with n-3 polyunsaturated fatty acids and vitamin E after myocardial infarction: results of the GISSIPrevenzione trial. Gruppo Italiano per lo Studio della Sopravvivenza nell'Infarto miocardico [published erratum in Lancet 2007; 369:106 and Lancet 2001;357:642]. Lancet 1999;354:447-55.

8. Yokoyama M, Origasa H, Matsuzaki M, et al. Effects of eicosapentaenoic acid on major coronary events in hypercholesterolaemic patients (JELIS): a randomised open-label, blinded endpoint analysis. Lancet 2007;369:1090-8.

9. Institute of Medicine. Dietary reference intakes for energy, carbohydrate, fiber, fat, fatty acids, cholesterol, protein, and amino 
acids (macronutrients). Washington (DC): National Academies Press; 2005:1-1331.

10. Cunnane SC. Problems with essential fatty acids: Time for a new paradigm? Prog Lipid Res 2003;42:544-68.

11. Cunnane SC, Guesnet P. Linoleic acid recommendations - a house of cards. Prostaglandins Leukot Essent Fatty Acids 2011;85: 399-402.

12. Do Canadian adults meet their nutrient requirements through food intake alone? Ottawa (ON): Health Canada; 2012. Available: www.hc-sc.gc.ca/fn-an/surveill/nutrition/commun/art-nutr -adult-eng.php\#a322 (accessed 2013 Feb. 8)

13. Mozaffarian D, Micha R, Wallace S. Effects on coronary heart disease of increasing polyunsaturated fat in place of saturated fat: a systematic review and meta-analysis of randomized controlled trials. PLoS Med 2010;7:e1000252.

14. Beck L. Which do I need in my diet: omega-3s or omega-6s? Globe and Mail [Toronto] 2013 Feb. 15.

15. Harris WS, Mozaffarian D, Rimm E, et al. Omega-6 fatty acids and risk for cardiovascular disease: a science advisory from the American Heart Association Nutrition Subcommittee of the Council on Nutrition, Physical Activity, and Metabolism; Council on Cardiovascular Nursing; and Council on Epidemiology and Prevention. Circulation 2009;119:902-7.

16. Aranceta J, Perez-Rodrigo C. Recommended dietary reference intakes, nutritional goals and dietary guidelines for fat and fatty acids: a systematic review. Br J Nutr 2012;107(Suppl 2):S8-22.

17. Ramsden CE, Hibbeln JR, Majchrzak SF, et al. n-6 fatty acidspecific and mixed polyunsaturate dietary interventions have different effects on CHD risk: a meta-analysis of randomised controlled trials. Br J Nutr 2010;104:1586-600.

18. Calder PC. The American Heart Association advisory on $n-6$ fatty acids: Evidence based or biased evidence? Br J Nutr 2010; 104:1575-6.

19. Canadian Nutrient File [database]. Ottawa $(\mathrm{ON})$ : Health Canada. Available: http://webprod3.hc-sc.gc.ca/cnf-fce (accessed 2013 Nov, 5).

20. Przybylski R. Canola oil: physical and chemical properties Winnipeg (MB): Canola Council of Canada. Available: www .canolacouncil.org/media/515239/canola_oil_physical_chemical _properties_1.pdf (accessed 2013 Nov. 5)
21. London SJ, Sacks FM, Caesar J, et al. Fatty acid composition of subcutaneous adipose tissue and diet in postmenopausal US women. Am J Clin Nutr 1991;54:340-5.

22. Garland M, Sacks FM, Colditz GA, et al. The relation between dietary intake and adipose tissue composition of selected fatty acids in US women. Am J Clin Nutr 1998;67:25-30.

23. Sun Q, Ma J, Campos H, et al. Comparison between plasma and erythrocyte fatty acid content as biomarkers of fatty acid intake in US women. Am J Clin Nutr 2007;86:74-81.

24. Chiuve SE, Rimm EB, Sandhu RK, et al. Dietary fat quality and risk of sudden cardiac death in women. Am J Clin Nutr 2012;96: 498-507.

25. Lucas M, Mirzaei F, O'Reilly EJ, et al. Dietary intake of n-3 and n6 fatty acids and the risk of clinical depression in women: a 10-y prospective follow-up study. Am J Clin Nutr 2011;93:1337-43.

26. Lucas M, Mirzaei F, O'Reilly EJ, et al. Relation entre n-3 et n-6 avec la depression clinique: resultats de la Nurses' Health Study. OLC 2011;18:181-7.

27. Production, supply and distribution online. Washington (DC) United States Department of Agriculture; 2012. Available: www.fas .usda.gov/psdonline/psdQuery.aspx (accessed 2013 Feb. 7).

28. Pan A, Chen M, Chowdhury R, et al. Alpha-linolenic acid and risk of cardiovascular disease: a systematic review and metaanalysis. Am J Clin Nutr 2012;96:1262-73.

29. Kromhout D, Giltay EJ, Geleijnse JM. n-3 fatty acids and cardiovascular events after myocardial infarction. $N$ Engl J Med 2010; $363: 2015-26$.

Affiliations: Department of Nutritional Sciences, Faculty of Medicine (Bazinet), University of Toronto, Toronto, Ont.; Division of Cardiac Surgery, Department of Surgery (Chu), Western University, London, Ont.; Lawson Health Research Institute (Chu), London, Ont.

Contributors: Both authors were responsible for the conception and design of the article, and the collection, analysis and interpretation of the data. Both of the authors drafted the manuscript, revised it for important intellectual content and approved the final version submitted for publication.

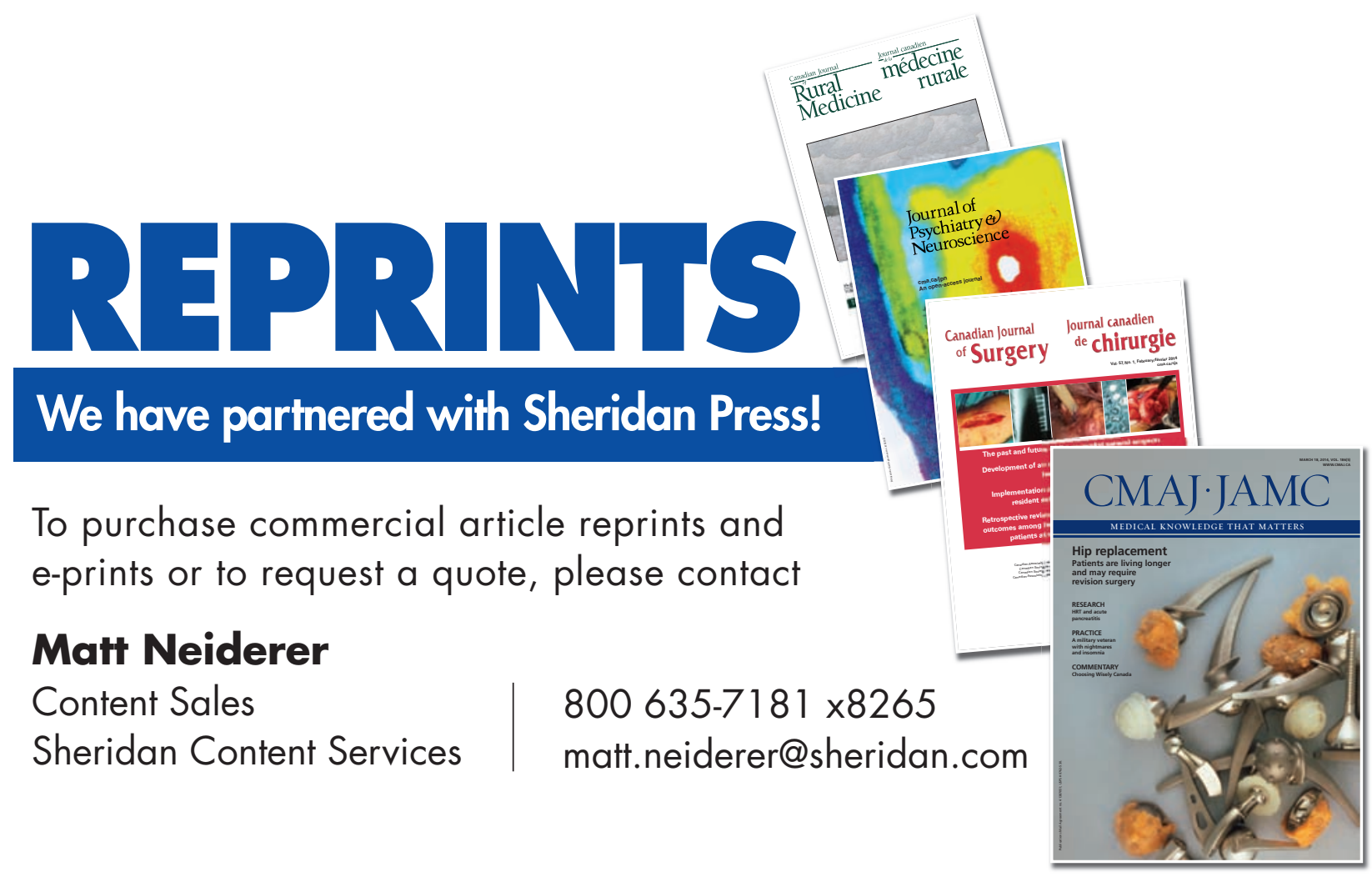

